\title{
Infinite Graphs with Finite 2-Distinguishing Cost
}

\author{
Debra Boutin \\ Hamilton College \\ Clinton, NY 13323, USA \\ dboutin@hamilton. edu
}

\author{
Wilfried Imrich \\ Montanuniversität Leoben \\ 8700 Leoben, Austria \\ imrich@unileoben.ac.at
}

Submitted: Apr 9, 2014; Accepted: Dec 4, 2014; Published: Dec 11, 2014

Mathematics Subject Classifications: 05C15, 05C25

\begin{abstract}
A graph $G$ is said to be 2-distinguishable if there is a labeling of the vertices with two labels such that only the trivial automorphism preserves the labels. Call the minimum size of a label class in such a labeling of $G$ the cost of 2-distinguishing $G$.

Within the class of connected, locally finite, infinite graphs, we show that those with finite 2-distinguishing cost are precisely the graphs with countable automorphism group. Further we show that, for such graphs, the cost is less than three times the size of a smallest determining set (a set which only the trivial automorphism fixes pointwise). Finally we show that graphs with linear growth rate $c$ have the even smaller upper bound of $c+1$ on their cost of 2-distinguishing.
\end{abstract}

Key words: Distinguishing number, Distinguishability, Automorphism, Determining set, Determining number, Infinite graph

\section{Introduction}

A labeling of the vertices of a graph $G$ with the integers $1, \ldots, d$ is called $d$-distinguishing if no nontrivial automorphism of $G$ preserves the labels. A graph is called d-distinguishable if it has a $d$-distinguishing labeling. This concept was introduced by Albertson and Collins in [2] and has spawned a wealth of results, in particular for finite graphs, but also for infinite ones.

For finite graphs it was shown that many infinite families of graphs have the property that all but finitely many members are 2-distinguishable; see [9]. Interestingly, in such cases the size of the smaller color class can be extremely small. For example the hypercube $Q_{2^{k}}$ of dimension $2^{k}$ can be 2 -distinguished by coloring $k+2$ vertices black and the others white; see [5]. 
There are also large classes of 2-distinguishable infinite graphs. In this paper, we consider the question of whether one of the two color classes can be finite, and how to obtain good bounds for the size of such a class.

To aid in addressing this question, we will call a label class in a 2-distinguishing labeling a distinguishing class. Following [4] we call the minimum size of a distinguishing class for a 2-distinguishable graph $\mathrm{G}$ the cost of 2-distinguishing $G$ and denote it by $\rho(G)$.

In the finite case it is natural to restrict the investigation to connected graphs. Thus the first natural extension to the infinite is to connected, locally finite, infinite graphs. That is, connected graphs that are infinite, and whose vertices have finite, but possibly arbitrarily large degrees. We show that such graphs $G$ have finite $\rho(G)$ if and only if $\operatorname{Aut}(G)$ is countable. Further we provide two general bounds for $\rho(G)$ and a sharper one for graphs of linear growth.

\section{Preliminaries}

Recall that the set stabilizer of $S \subseteq V(G)$, denoted $\operatorname{SetStab}(S)$, is the set of all $\varphi \in \operatorname{Aut}(G)$ for which $\varphi(x) \in S$ for all $x \in S$. In this case we say that $S$ is invariant under $\varphi$, or $\varphi$ preserves $S$, and we write $\varphi(S)=S$. The point stabilizer of $S$, denoted $\operatorname{PtStab}(S)$ is the set of all $\varphi \in \operatorname{Aut}(G)$ for which $\varphi(x)=x$ for all $x \in S$.

A labeling of the vertices of a graph $G$ with the integers $1, \ldots, d$ is called a $d$ distinguishing labeling if no non-trivial automorphism of $G$ preserves the labels. A graph is called $d$-distinguishable if it has a $d$-distinguishing labeling. If a graph is 2-distinguishable, call a color class in a 2-distinguishing labeling a distinguishing class for $G$. The minimum size of a distinguishing class for a 2-distinguishable graph $G$ is called the cost of 2 -distinguishing $G$ and is denoted by $\rho(G)$.

A significant tool used in this work is a determining set [3] (or equivalently, a base of the automorphism group action). A determining set $S$ has the property that whenever $\varphi, \psi \in \operatorname{Aut}(G)$ so that $\varphi(x)=\psi(x)$ for all $x \in S$, then $\varphi=\psi$. Thus every automorphism of $G$ is uniquely determined by its action on the vertices of a determining set. Equivalently, a determining set is a set of vertices $S$ with $\operatorname{PtStab}(S)=\{\mathrm{id}\}$. The determining number of the graph $G$, denoted by $\operatorname{Det}(G)$, is the minimum size of a determining set of $G$.

Note that for $F \subseteq V(G)$, an automorphisms $\varphi \in \operatorname{SetStab}(F)$ can be thought of as a permutation in $\operatorname{Sym}(F)$ by restricting the action of $\varphi$ to $F$, denoted $\varphi \mid F$. Thus we have a natural map $\Psi: \operatorname{SetStab}(F) \rightarrow \operatorname{Aut}(G) \mid F \leqslant \operatorname{Sym}(F)$. Note that this map is injective if and only if $F$ is a determining set for $G$. In such a case, we get that $\operatorname{SetStab}(F) \cong \operatorname{Aut}(G) \mid F$.

Albertson and Boutin showed in [1] that a graph is $d$-distinguishable if and only if it has a determining set that is $(d-1)$-distinguishable. In particular, such a determining set is a distinguishing class for a 2-distinguishable graph $G$. Thus, a graph is 2-distinguishable if and only if it has a determining set $S$ for which $\operatorname{Set} \operatorname{Stab}(S)=\operatorname{PtStab}(S)=\{$ id $\}$. In such a case, the determining set and its complement provide the two necessary label classes for a 2-distinguishing labeling. Thus in particular, the cost of 2-distinguishing a graph $G$ is bounded below by the size of a smallest determining set. 
The motion of an automorphism $\varphi \in \operatorname{Aut}(G)$, denoted $\mathrm{m}(\varphi)$, is the number of vertices moved by $\varphi$. The motion of the automorphism group, denoted $\mathrm{m}(G)$, is the minimum motion of the non-trivial elements of $\operatorname{Aut}(G)$.

Throughout this paper let $\Gamma$ denote the class of infinite, connected, locally finite graphs. For such graphs Halin proved the following result, which is foundational for the work that follows.

Theorem 2.1 [8] (Halin, 1973) A connected, locally finite infinite graph $G$ has uncountable $\operatorname{Aut}(G)$ if and only if for every finite $F \subset V(G)$ there exists a non-trivial automorphism $\varphi$ of $G$ such that $\varphi(v)=v$ for each $v \in F$.

In other words, $G \in \Gamma$ has uncountable automorphism group if and only if it does not have a finite determining set. Therefore, a 2-distinguishable graph $G$ with uncountable automorphism group has infinite $\rho(G)$. We are thus interested in graphs $G$ of $\Gamma$ whose automorphism group is not uncountable. We first consider those that have infinite automorphism group. For them we have the following extension of Theorem 2.1.

Theorem $2.2[9]$ (Imrich, Smith, Tucker, Watkins, 2014) If $G \in \Gamma$ so that $\aleph_{0} \leqslant|\operatorname{Aut}(G)|$ $<2^{\aleph_{0}}$, then $\operatorname{Det}(G)<\aleph_{0},|\operatorname{Aut}(G)|=\aleph_{0}, m(G)=\aleph_{0}$, and $G$ is 2-distinguishable. This holds independently of the Continuum Hypothesis.

There is yet another consequence of Theorem 2.1 that is folklore. It says that the vertex stabilizers of graphs $G \in \Gamma$ are finite if $\operatorname{Aut}(G)$ is countably infinite. This follows, for example, from the slightly more general Corollary 3.10 from [9]. Below we state and prove a related result that invokes neither Theorem 2.1 nor Theorem 2.2, but needs the following definition: The set of vertices $u \in V(G)$ for which $d(u, v) \leqslant n$ is called ball of radius $n$ centered at $v$, and denoted $B_{v}(n)$. For later reference we also define the sphere $S_{v}(n)$ of radius $n$ centered at $v$ as the set of vertices $u \in V(G)$ for which $d(u, v)=n$.

Lemma 2.3 If $G \in \Gamma$ has finite determining set, then the vertex stabilizers of $G$ are finite.

Proof Suppose $B$ is a finite determining set for $G$ and $\operatorname{PtStab}(v)$ is infinite. Since $B$ is finite, we may choose $k$ so that $B \subseteq B_{v}(k)$. Hence, the orbit $C=\operatorname{PtStab}(v)(B)$ of $B$ under $\operatorname{PtStab}(v)$ is a subset of $B_{v}(k)$ and thus is finite. Because the infinite group $\operatorname{PtStab}(v)$ stabilizes the finite set $C$ setwise, there must be at least two different elements of $\operatorname{PtStab}(v)$ whose actions are identical on $C$, and thus also on $B$. This contradicts the choice of $B$ as a determining set.

\section{Countable Automorphism Group}

By Theorem 2.2 the automorphism groups of graphs $G$ in $\Gamma$ that satisfy $\aleph_{0} \leqslant|\operatorname{Aut}(G)|<$ $2^{\aleph_{0}}$ are countable. Moreover, such graphs have infinite motion, are 2-distinguishable, and have finite determining sets. Below we show that such graphs $G$ have finite 2distinguishing cost and give two bounds for $\rho(G)$. 
Lemma 3.1 If $G \in \Gamma$ with infinite automorphism group and $\operatorname{Det}(G)=n<\aleph_{0}$, then $\rho(G)$ is finite and satisfies the inequality

$$
\rho(G) \leqslant n+n !-1 .
$$

Proof Let $F$ be a minimum determining set for $G$. Since $F$ is a determining set, as discussed in $\operatorname{Section} 2, \operatorname{SetStab}(F) \cong \operatorname{Aut}(G) \mid F \leqslant \operatorname{Sym}(F)$. Since $F$ is finite, so is $\operatorname{Sym}(F)$, and hence so is $\operatorname{SetStab}(F)$. Let $\operatorname{SetStab}(F)=\left\{\mathrm{id}=\alpha_{0}, \alpha_{1}, \alpha_{2}, \ldots, \alpha_{k}\right\}$. If $|F|=1$, then $k=0$. We can thus assume that $|F|>1$.

Color all elements of $F$ black and consider $\alpha_{1}$. By Theorem 2.2, $G$ has infinite motion. In other words, every nontrivial automorphism moves infinitely many vertices. Since $\alpha_{1}$ moves infinitely many vertices, there is a vertex $v_{1}$, moved by $\alpha_{1}$, whose distance $d\left(v_{1}, F\right)$ from $F$ is larger than the diameter of $F$. Color it black. Similarly there is a vertex $v_{2}$, moved by $\alpha_{2}$, with $d\left(v_{2}, F\right)>d\left(v_{1}, F\right)+\operatorname{diam}(F)$. Color it black as well. Similarly construct the vertices $v_{3}$ to $v_{k}$ and color them black.

Suppose that $\varphi$ preserves the set of black vertices. Since each of $v_{1}, \ldots, v_{k}$ is farther from $F$ than any pair of vertices in $F$ is, $\varphi$ must preserve $F$. Thus $\varphi \in \operatorname{SetStab}(F)$. Thus either $\varphi=i d$ or $\varphi=\alpha_{i}$ for some $i \in[k]$. Recall that, for each $i \in[k]$, since $v_{i}$ is the only black vertex of distance $d\left(v_{i}, F\right)$ from $F$, we have that $\varphi\left(v_{i}\right)=v_{i}$. Since $v_{i}$ is moved by $\alpha_{i}$, we infer $\varphi \neq \alpha_{i}$. Thus $\varphi=i d$ and the set of black vertices is a distinguishing class. Clearly its size is $|\operatorname{SetStab}(F)|-1+|F| \leqslant n !+n-1$. Hence, $\rho(G)<\aleph_{0}$.

Interestingly this crude bound is sharp for the 2 -sided infinite path $P_{\aleph_{0}}$. To see this, observe that no single vertex of $P_{\aleph_{0}}$ can be a determining set, but that the set of endpoints of any edge is a determining set. By the lemma, $\rho\left(P_{\aleph_{0}}\right) \leqslant 3$. It is easy to see that $\rho\left(P_{\aleph_{0}}\right)$ cannot be 1 or 2 . Hence $\rho\left(P_{\aleph_{0}}\right)=3$ and the bound is sharp.

A better bound is the following, which we formulate as theorem. It uses a strong result of Cameron, Solomon and Turull [6], which asserts that the largest length of a chain of subgroups of the symmetric group on $n$ elements is $\left\lceil\frac{3 n}{2}\right\rceil-b(n)-1$, where $b(n)$ denotes the number of $1 \mathrm{~s}$ in the base-2 representation of $n$.

Theorem 3.2 If $G \in \Gamma$ with infinite automorphism group and $\operatorname{Det}(G)=n<\aleph_{0}$, then $\rho(G) \leqslant\left\lceil\frac{5 n}{2}\right\rceil-b(n)-1$, where $b(n)$ denotes the number of $1 s$ in the base-2 representation of $n$.

Proof Let $F$ be a minimum determining set for $G$. Then $n=|F|=\operatorname{Det}(G)$. Let $k, \alpha_{i}, v_{i}$ for $i \in[k]$ be as in the proof of Lemma 3.1. As shown above, coloring all elements of $F$ black breaks all automorphisms of $G$ that do not preserve $F$. The automorphisms that preserve $F$ form a (not necessarily proper) subgroup $A_{0}$ of $\operatorname{Sym}(F)$. Coloring $v_{1}$ black leaves a subgroup $A_{1}<A_{0}$ of still unbroken elements of $\operatorname{Aut}(G)$. Using the notation $A_{i}$ for the group that preserves the set $F \cup\left\{v_{1}, \ldots, v_{i}\right\}$ we thus arrive at a chain of subgroups

$$
\{\operatorname{id}\}=A_{k}<A_{k-1}<\cdots<A_{0}
$$

of length $k$. By $[6], k \leqslant\left\lceil\frac{3 n}{2}\right\rceil-b(n)-1$. Because we colored $k+n$ vertices black the result follows. 
Again, this bound is sharp for $P_{\aleph_{0}}$. From $\operatorname{Det}\left(P_{\aleph_{0}}\right)=2$ we infer $\rho\left(P_{\aleph_{0}}\right) \leqslant\lceil 5 \cdot|F| / 2\rceil-$ $b(|F|)-1=5-1-1=3$, which we know is the cost of 2-distinguishing $P_{\aleph_{0}}$.

We now combine some of the above results for our main theorem.

Theorem 3.3 Let $G \in \Gamma$ with infinite automorphism group. Then $\rho(G)$ is finite if and only if $\operatorname{Aut}(G)$ is countable.

Proof Let $G$ be a graph in $\Gamma$ with finite $\rho(G)$. Since a distinguishing class is necessarily a determining set, we can conclude that $G$ has a finite determining set. Then, by Theorem 2.1, $\operatorname{Aut}(G)$ cannot be uncountable. Thus it is countable.

Suppose $G \in \Gamma$ has countable automorphism group. By Theorem 2.2, $G$ is both 2distinguishable and has a finite determining set. By using either Lemma 3.1 or Theorem 3.2 we conclude that $\rho(G)$ is finite.

\section{Linear Growth}

For graphs of linear growth we can obtain an even better bound for the cost of 2distinguishing. We begin with a few remarks about graphs of linear growth.

A connected, locally finite, infinite graph $G$ is said to have linear growth if there exists a vertex $v$ and a constant $c$, such that $\left|B_{v}(n)\right| \leqslant c n$, for all $n \in \mathbb{N}$. The definition is independent of the choice of $v$, but $c$ may have to be replaced by a different constant if $v$ is changed.

Notice that $B_{v}(n)=\cup_{i=0}^{n} S_{v}(i)$ and that the growth is obviously linear if $\left|S_{v}(n)\right| \leqslant c$ for all $n$. Nonetheless, it is possible that infinitely many spheres have more than $c$ elements. Moreover, the size of the spheres need not even be bounded. We leave it to the reader to construct examples.

More important for us is the fact that there must be infinitely many spheres of size at most $c$. To see this, suppose only $m<\aleph_{0}$ of the spheres around $v$ contain at most $c$ vertices. Consider $B_{v}(\mathrm{~cm}+1)=\cup_{i=0}^{c m+1} S_{v}(i)$. The $m$ spheres of size at most $c$ contain at least one vertex each, and thus in total contain at least $m$ vertices. The $(c-1) m+1$ spheres with more than $c$ vertices contain at least $c+1$ vertices each, and thus contain at least $(c+1)(c m+1-m)$ in total. Thus $\left|B_{v}(c m+1)\right|=\cup_{i=0}^{c m+1}\left|S_{v}(i)\right| \geqslant m+((c-1) m+1)(c+1)=$ $c(c m+1)+1$ which contradicts the assumption on the growth of $G$. Thus there are an infinite number of spheres of size at most $c$. Because $c$ is finite, infinitely many of these spheres must have the same size.

Interestingly, for graphs $G \in \Gamma$ with linear growth and infinite automorphism group, $\mathrm{m}(G)=\aleph_{0}$ if and only if $|\operatorname{Aut}(G)|=\aleph_{0}$. That $|\operatorname{Aut}(G)|=\aleph_{0}$ implies infinite motion follows from Theorem 2.2. The reverse implication is not hard to show. In [7] it is attributed to [9], but only the consequence that $G$ is 2-distinguishable is mentioned there. As that paper contains no proof of it, we include one here for the sake of completeness. The proof uses the following lemma, which is similar to Lemma 2.4 in [7]. 
Lemma 4.1 Suppose that $G$ is a connected, locally finite graph and $\operatorname{Aut}(G)$ has infinite motion. If $\alpha, \beta \in \operatorname{PtStab}(v)$ and $\alpha\left|S_{v}(n)=\beta\right| S_{v}(n)$ for some $n>0$, then $\alpha \mid B_{v}(n)=$ $\beta \mid B_{v}(n)$. Furthermore, if $\alpha, \beta \in \operatorname{PtStab}(v)$ have distinct actions on $S_{v}(n)$, then they have distinct actions on all $S_{v}(m)$ where $m>n$.

Proof Define the mapping $\gamma$ on $V(G)$ by $\gamma(u)=u$ for all $u$ with $d(v, u) \geqslant n$ and $\gamma(u)=\alpha \beta^{-1}(u)$ for all $u$ with $d(v, u)<n$. Clearly, $\gamma$ is one-to-one and onto and preserves adjacency, so $\gamma$ is an automorphism. Since $\operatorname{Aut}(G)$ has infinite motion, but $\gamma$ moves at most finitely many vertices (those inside $\left.B_{v}(n-1)\right), \gamma=i d$. This proves the first assertion. The second assertion easily follows from the first.

Lemma 4.2 Let $G \in \Gamma$ be a graph of linear growth with infinite automorphism group. Then $\operatorname{Aut}(G)$ is countable if and only if $G$ has infinite motion.

Proof By Theorem 2.2 we only have to show that infinite motion implies that $\operatorname{Aut}(G)$ is countable. For distinct $\alpha, \beta \in \operatorname{PtStab}(v)$ there is some $k$ so that $\alpha, \beta$ differ in their action on $S_{v}(k)$. If $\operatorname{PtStab}(v)$ is finite then there exists some $k$ so that all pairs of automorphisms in $\operatorname{PtStab}(v)$ differ in their actions on the finite set $B_{v}(k)$. In such a case $\operatorname{Det}(G)$ is finite and thus by Theorem 2.1, Aut $(G)$ is countable. Thus it is sufficient to prove that point stabilizers in $G$ are finite. Suppose $\operatorname{PtStab}(v)$ is infinite. Since $G$ has linear growth, by our previous argument we know that there are infinitely many spheres with center $v$ that have size at most $c$. Since $\operatorname{PtStab}(v)$ is infinite, we may consider $c !+1$ distinct elements of $\operatorname{PtStab}(v)$. Any pair of these must act distinctly on some sphere $S_{v}(n)$, and thus by Lemma 4.1, on all spheres $S_{v}(m)$ for $m>n$. Hence, there must be a sphere of size $c$ on which all $c !+1$ automorphisms have distinct action. But this is impossible since there are at most $c$ ! distinct actions on a set of size $c$. Thus $|\operatorname{PtStab}(v)|<\aleph_{0}$. In particular, if $G \in \Gamma$ has infinite motion and linear growth rate $c$ from vertex $v$, then then $|\operatorname{PtStab}(v)| \leqslant b$ ! where $b \leqslant c$ is the minimum sphere size that occurs infinitely often.

The part of the lemma which asserts that linear growth and infinite motion imply countability of the automorphisms group is based on an observation by T. Tucker ${ }^{1}$.

Theorem 4.3 Let $G$ be a graph with countably infinite automorphism group and linear growth. If $\left|B_{v}(n)\right| \leqslant c n$ for a fixed $v \in V(G)$ and $c \in \mathbb{R}$, then $\rho(G) \leqslant c+1$.

Proof Notice first that since $G$ has a countable automorphism group, by Theorem 2.2, it has infinite motion. By our previous argument, there are infinitely many spheres of size at most $c$ centered at $v$. Denote these by $S_{i}=S_{v}\left(n_{i}\right)$, for $n_{1}<n_{2}<\cdots$. Since $c$ is finite, infinitely many of these spheres must have the same size. Without loss of generality we can assume that the $S_{i}$ already have the same size, say $d$.

Note that for $j<i$, the minimal distance between an arbitrary vertex in $S_{i}$ and one in $S_{j}$ is $n_{i}-n_{j}$ and the maximal distance is $n_{i}+n_{j}$. Hence, if we choose the $n_{i}$ such that

$$
n_{i}>2 \sum_{j<i} n_{j}
$$

\footnotetext{
${ }^{1}$ Private communication.
} 
then for $j<i$ the distance between an arbitrary vertex in $S_{i}$ and one in $S_{j}$ is larger than the maximal distance of any two vertices in $\cup_{j<i} S_{j}$.

By Lemma 4.1, since $G$ has infinite motion, every non-identity element of $\operatorname{PtStab}(v)$ acts non-trivially on all but finitely many spheres. As PtStab $(v)$ is finite by Lemma 2.3, without loss of generality, we can assume that $\operatorname{PtStab}(v) \backslash\{$ id $\}$ acts non-trivially on all spheres $S_{i}$.

For the sequel we wish to remind the reader that we have chosen the notation such that mappings act on the left.

For each $i$ greater than 1 , fix an arbitrary bijection $\phi_{i}$ from $S_{1}$ to $S_{i}$. Let $\phi_{1}=i d$ on $S_{1}$. For each $i$, denote by $A_{i}$ the group $\phi_{i}^{-1}\left(\operatorname{PtStab}(v) \mid S_{i}\right) \phi_{i}$. By definition, each of the $A_{i}$ is a subgroup of $\operatorname{Sym}\left(S_{1}\right)$. Further, consider an arbitrarily chosen $\alpha \in \operatorname{PtStab}(v)$ and the infinite set of permutations $\left\{\beta_{i}=\phi_{i}^{-1}\left(\alpha \mid S_{i}\right) \phi_{i}\right\} \subseteq \operatorname{Sym}\left(S_{1}\right)$. As $\operatorname{Sym}\left(S_{1}\right)$ has only finitely many elements, infinitely many of the $\beta_{i}$ must identical. Let $J$ be the set of indices for which this is the case. Notice, that this set need not include 1 . Let $j_{0}$ be the smallest of these indices. Clearly, for $j \in J$ all

$$
\gamma_{j}=\phi_{j_{0}} \phi_{j}^{-1}\left(\alpha \mid S_{j}\right) \phi_{j} \phi_{j_{0}}^{-1}
$$

are identical on $S_{j_{0}}$, and

$$
\gamma_{j_{0}}=\phi_{j_{0}} \phi_{j_{0}}^{-1}\left(\alpha \mid S_{j_{0}}\right) \phi_{j_{0}} \phi_{j_{0}}^{-1}=\alpha \mid S_{j_{0}} .
$$

Notice that the $\phi_{j} \phi_{j_{0}}^{-1}$ are bijections from $S_{j_{0}}$ to $S_{j}$. Hence, we can assume without loss of generality that $J$ is $\mathbb{N}$, in other words that all $\beta_{i}$ are identical and that $\beta_{1}=\alpha \mid S_{1}$.

$\operatorname{Because} \operatorname{PtStab}(v)$ is finite, we can, proceeding successively, suppose that for any element $\psi \in \operatorname{PtStab}(v)$ the equalities

$$
\psi \mid S_{1}=\phi_{i}^{-1}\left(\psi \mid S_{i}\right) \phi_{i}
$$

hold.

Again we can assume without loss of generality that this is already the case for the chosen $S_{i}$.

Suppose $\alpha \in \operatorname{PtStab}(v)$ fixes a vertex $\phi_{i}(u)$ in $S_{i}$. Then $\alpha(u)=\phi_{1}^{-1} \alpha \phi_{1}(u)=$ $\phi_{i}^{-1} \alpha \phi_{i}(u)=\phi_{i}^{-1}\left(\alpha\left(\phi_{i}(u)\right)\right)=\phi_{i}^{-1} \phi_{i}(u)=u$. That is, if $\alpha \in \operatorname{PtStab}(v)$ fixes $\phi_{i}(u)$ in $S_{i}$ then $\alpha$ fixes $u$ in $S_{1}$.

Finally, let $v_{1}, v_{2}, \ldots v_{d}$ be the vertices of $S_{1}$. Color the vertices of $X=\left\{v, \phi_{1}\left(v_{1}\right)\right.$, $\left.\phi_{2}\left(v_{2}\right), \ldots, \phi_{d}\left(v_{d}\right)\right\}$ black. Color all other vertices in the graph white. Suppose $\alpha \in \operatorname{Aut}(G)$ preserves color classes. By our choice of distances between the spheres $S_{1}, \ldots, S_{d}$, within the set $X v$ is uniquely identified by its distances to the other vertices.

Thus $\alpha \in \operatorname{PtStab}(v)$. Since $\alpha$ must preserve distance to $v$, again by the way distances were chosen, $\alpha$ cannot interchange vertices of $\left\{\phi_{1}\left(v_{1}\right), \ldots, \phi_{d}\left(v_{d}\right)\right\}$. Thus $\operatorname{PtStab}(v)$ fixes all $\phi_{i}\left(v_{i}\right)$, and hence all $v_{i}$. Recall that the spheres $S_{i}$ were chosen so that every non-trivial automorphism in $\operatorname{PtStab}(v)$ acts non-trivially on each sphere. Thus if $\alpha$ is non-trivial, its action on $S_{1}$ must be nontrivial, but this is not possible, since it fixes all elements of $S_{1}$. So $\alpha$ is the identity. 
To see that the bound is sharp, consider $P_{\aleph_{0}}$. It has linear growth with $c=2$. We know that it can be distinguished by just three black vertices, but not by two. Hence $\rho\left(P_{\aleph_{0}}\right)=3$, which is the bound given by the theorem.

\section{Finite Automorphism Group}

Suppose the automorphism group of $G \in \Gamma$ is finite. There are two types of automorphisms, those with finite motion and those with infinite motion. Clearly the ones with finite motion form a subgroup of (the finite group) Aut $(G)$, say $B$. Define $W \subseteq V(G)$ to be the orbits under $B$ of all vertices moved by $B$. Since $B$ is finite, and all motion in $B$ is finite, $W$ is also finite. Further $W$ is stabilized setwise by $B$ while elements of $V(G) \backslash W$ are fixed by $B$.

Consider a distinguishing 2-coloring of $V(G)$ with the color classes $X_{1}$ and $X_{2}$ (white and black). We set $Y_{1}=X_{1} \cap W$ and $Y_{2}=X_{2} \cap W$. Since $X_{1}, X_{2}$ distinguished the action of $\operatorname{Aut}(G), Y_{1}, Y_{2}$ distinguish the action of $B$, independent of the colors of the elements of $V(G) \backslash W$.

Let $\left\{\alpha_{1}, \alpha_{2}, \ldots, \alpha_{k}\right\}$ be the elements of infinite motion in $\operatorname{Aut}(G)$. To break the symmetries of each of these, we first color all elements of $V(G) \backslash W$ white and then use the methods of Lemma 3.1 to choose vertices $v_{1}, v_{2}, \ldots, v_{k}$ to color black.

Clearly $X_{2} \cup\left\{v_{1}, v_{2}, \ldots, v_{k}\right\}$ is finite, and hence also $\rho(G)$. We have thus proved the following lemma.

Theorem 5.1 Let $G$ be a 2-distinguishable graph in $\Gamma$. If $\operatorname{Aut}(G)$ is finite, then $\rho(G)$ is also finite.

\section{Acknowledgements}

Wilfried Imrich was partially supported by ARRS Slovenia within the EUROCORES Programme EUROGIGA/GReGAS of the European Science Foundation.

The authors also wish to thank Simon Mark Smith and Thomas W. Tucker for their careful attention which helped improve the clarity of the paper.

\section{References}

[1] M. O. Albertson, D. L. Boutin, Using determining sets to distinguish Kneser graphs, Electron. J. Combin., 14(1) (2007), \#R20, 9 pp.

[2] M. O. Albertson and K. L. Collins, Symmetry breaking in graphs, Electron. J. Combin., 3(1) (1996), \#R18, 17 pp.

[3] D. L. Boutin, Identifying graph automorphisms using determining sets, Electron. J. Combin., 13(1) (2006), \#R78, 12 pp.

[4] D. L. Boutin, Small label classes in 2-distinguishing labelings. Ars Math. Contemp., 1 (2008), no. 2, 154-164. 
[5] D. L. Boutin, The Cost of 2-Distinguishing Selected Kneser Graphs and Hypercubes, J. Combin. Math. Combin. Comput., 85 (2013), 161-171.

[6] P. J. Cameron, R. Solomon and A. Turull, Chains of subgroups in the symmetric group, J. Algebra, 127 (1989), no. 2, 340-352.

[7] J. Cuno, W. Imrich and F. Lehner, Distinguishing graphs with infinite motion and nonlinear growth, Ars Math. Contemp., 7 (2014), no. 1, 201-213.

[8] R. Halin, Automorphisms and endomorphisms of infinite locally finite graphs, Abh. Math. Sem. Univ. Hamburg, 39 (1973), 251-283.

[9] W. Imrich, S. M. Smith, T. W. Tucker, and M. E. Watkins, Infinite motion and the distinguishing number of graphs and groups, J. Algebraic Comb., doi:10.1007/s10801-014-0529-2. 